\title{
UNLIMITED POINT ALGORITHM FOR OPF PROBLEMS
}

\author{
Giorgio Tognola \\ Giorgio.Tognola@eeh.ee.ethz.ch \\ Rainer Bacher \\ Rainer.Bacher@eeh.ee.ethz.ch \\ Swiss Federal Institute of Technology (ETH), CH 8092 Zürich, Switzerland
}

\begin{abstract}
A non-linear Optimal Power Flow (OPF) algorithm is presented which allows to solve the KarushKuhn-Tucker (KKT) optimality conditions using a pure Newton-Raphson solution procedure. The method is similar to interior point algorithms. However, due to a simple transformation, the variable space becomes unlimited (Unlimited Point) and variables do not need to be forced to stay within the feasible region during all OPF iterations as is the case for interior point algorithms. As a consequence only a pure Newton-Raphson iterative process to algebraically transformed KKT conditions is applied. The algorithm has been successfully applied to various networks up to size 700 buses with the active power loss objective function.
\end{abstract}

Keywords - Optimal Power Flow, Interior Point, Newton-Raphson, Optimality Conditions

\section{Introduction}

The mathematical formulation of the Optimal Power Flow (OPF) problem is a well known optimization problem. In general mathematical terms is can be formulated as follows:

$\begin{array}{ll}\text { Minimize } & \mathcal{F}(\mathbf{x}) \\ \text { subject to } & \mathbf{g}(\mathbf{x})=\mathbf{0} \\ \text { and } & \mathbf{h}(\mathbf{x}) \leq \mathbf{0}\end{array}$

An optimal solution to this problem must satisfy the Karush-Kuhn-Tucker optimality conditions. The optimality conditions for (1) can be derived by formulating the Lagrangian function $\mathcal{L}$ :

$$
\mathcal{L}=\mathcal{F}(\mathbf{x})+\lambda^{T} \mathbf{g}(\mathbf{x})+\mu^{T} \mathbf{h}(\mathbf{x})
$$

The Karush-Kuhn-Tucker (KKT) theorem [1] says that if $\widehat{\mathbf{x}}$ is the relative extremum of $\mathcal{F}(\mathbf{x})$ which satisfies at the same time all constraints of (1), vectors $\widehat{\lambda}, \widehat{\mu}$ must exist which satisfy the following system of equations:

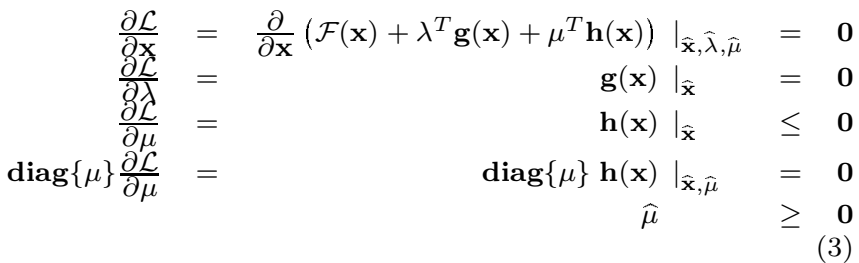

Two algorithmic solution classes exist for (1): Class A approaches [2, 3] combine the strengths of AC power flow solution and standard LP/QP (Linear/Quadratic programming) to approximated problem formulations of (1). They determine the optimal solution of the original nonlinear optimization problem (1) iteratively. Class B algorithms [2,3] solve the optimality conditions of (1), i.e. (3): Due to the fact that (3) can be highly non-linear, the solution of (3) is done by iteratively solving approximations to slightly modified versions of (3).

In this paper we concentrate on the solution of the OPF problem by the class B approach. Some of the concepts presented here can also be applied to the LP/QP optimization problem part of class A algorithms.

\subsection{OPF Class B approaches}

In contrast to class A OPF algorithms, class B OPF algorithms do not approximate the original OPF problem (1): They solve the KKT-optimality conditions (3). The main problem is the high non-linearity and dimensionality of the equality and inequality functions which must be satisfied. The complementarity condition (fourth condition in (3)) together with the need to have positive $\mu$-Lagrange multipliers are particularly difficult to handle.

Solution methods for (3) have in common that they use in some form a method similar to a Newton-Raphson method. This method, however, can be applied only to a well determined set of non-linear equations. In order not to loose the strengths of the Newton-Raphson solution methodology two solution approaches can be seen in the literature: The first is called class B.1, the second B.2.

\subsubsection{Class B.1: Penalty approach}

Class B.1 is the so-called Newton-approach [4] where the objective function is augmented by adding the inequality constraints as penalty terms $\left(\mathbf{w}^{T} \mathbf{h}^{\mathbf{2}}(\mathbf{x})\right)$ :

$$
\begin{array}{ll}
\mathcal{F}(\mathbf{x}) & :=\mathcal{F}(\mathbf{x})+\mathbf{w}^{T} \mathbf{h}^{2}(\mathbf{x}) \\
\mathbf{g}(\mathbf{x}) & =0
\end{array}
$$

In (4) the term $\mathbf{h}^{\mathbf{2}}(\mathbf{x})$ indicates that each individual element of $h(x)$ is squared. 
For such an optimization problem the KKT-conditions represent a well determined set of equations which can be solved with a Newton-Raphson solution method. If one could know beforehand which inequality constraints are binding at the optimal solution, very high weights $w_{i}$ would be used for the corresponding binding $h_{i}^{2}(x)$ - terms. Those inequality constraints which are not binding would ideally get a weight $w_{i}$ of zero.

The difficulty is that in general one cannot assume that the binding constraints are knowns beforehand. Thus, solution algorithms have built-in heuristic logic to dynamically determine from a Newton-Raphson iteration to the next how to set the weights. The trick is to select how hard (large $w_{i}$ ) or how soft (small $w_{i}$ ) the inequality constraints are modelled.

\subsubsection{Class B.2: Interior point}

Interior point (IP) algorithms have their origin in the solution of LP problems $[5,6]$. Today the so-called primaldual solution method seems to be the favorite implementation. This method combines both the primal and the dual LP problems into one single problem formulation. The class of primal-dual interior point algorithms use a modified version of a Newton-Raphson solution algorithm. The modification of a Newton-Raphson includes the following points which are common to all interior point algorithms: 1. Functional inequalities which are limited from above are converted to equality constraints by introducing slack variables $z$ which are limited from below.

$$
\mathbf{h}(\widehat{\mathbf{x}})+\widehat{\mathbf{z}}=0
$$

with

$$
\widehat{\mathbf{z}} \geq \mathbf{0}
$$

2. The iterative solution must start from an initial feasible solution for all limited variables including the slack variables.

3. Mathematicians have shown that the LP problem will converge to the optimal solution when a) a logarithmic barrier function term is added to the original objective function,

$$
\begin{array}{ll}
\mathcal{F}(\mathbf{x}) & :=\mathcal{F}(\mathbf{x})+\zeta \sum_{i}\left(\log _{e} z_{i}\right) \\
\mathbf{g}(\mathbf{x}) & =0 \\
\mathbf{h}(\mathbf{x})+\mathbf{z} & =0 \\
\mathbf{z} & \geq \mathbf{0}
\end{array}
$$

when b) the KKT-optimality conditions are formulated for this modified optimization problem, c) these KKToptimality conditions are solved using a Newton-Raphson procedure with step length control to remain with all limited variables within the feasible space and d) the barrier factor $\zeta$ is gradually decreased toward zero following heuristic rules.

The step length control and the rules to choose the decrease of the barrier parameter $\zeta$ are heuristic and are the major drawback of interior point methods.

Recently, the original LP based interior point methodology has been generalized to the class of linear complementarity problems [7] as follows:

$$
\begin{array}{ll}
\mathbf{y} & =\mathbf{M x}+\mathbf{q} \\
x_{i} y_{i} & =0 \forall i(i=1 \ldots n) \\
\mathbf{x} & \geq \mathbf{0} \\
\mathbf{y} & \geq \mathbf{0}
\end{array}
$$

with $\operatorname{dim}[\mathbf{M}]=n \cdot n, \mathbf{q} \in \mathcal{R}^{n}, \mathbf{x} \in \mathcal{R}^{n}$ and $\mathbf{y} \in \mathcal{R}^{n}$.

In [7] and [8] it is shown that the solution to this problem includes many versions of the primal-dual LP and QP problem interior point solution methods provided the matrix $M$ is a positive semidefinite matrix. Equation (8) does not include the barrier parameter of the interior point methodology. It is only the solution process for (8) which leads to the use of the barrier parameter. This is a distinct conceptual difference from the interior point methodology and will be used in the approach of this paper. It is also important to understand that, when adding the logarithmic penalty terms to an LP or QP objective function, the barrier parameter comes into (8) only in the terms $x_{i} y_{i}=\zeta \forall i \quad(i=1 \ldots n)$. (8) describes the optimality conditions at the optimal solution where $\zeta$ must be zero.

Primal-Dual interior point based solution methods have been applied to the KKT-conditions (3) of non-linear OPF-problem formulations [9, 10, 11, 12, 13, 14, 15]. All these approaches solve a linear system of equations per Newton-Raphson step. Each of these linear systems is conceptually similar to (8). From iteration to iteration, however, parts of the matrix $\mathbf{M}$ and the vector $\mathbf{q}$ change. All methods use heuristics to decrease the barrier parameter $\zeta$ from one iteration to the next; all methods use step length control to remain with all inequality constrained variables in the feasible space.

In the following a new approach is presented which takes advantage of the strenghts of both the "Newton approach" and the primal-dual interior point algorithms, while avoiding the main disadvantages of these methods: The problem of determining the barrier parameter (IP), the weights $w_{i}$ (Newton method) and the problem of satisfying feasibility after each Newton-Raphson step (IP).

\section{Unlimited point algorithm}

\subsection{Transformed KKT optimality conditions}

In this section the general non-linear OPF optimization problem is solved by an integrated method. The solution to the problem is based exclusively on a Newton-Raphson solution algorithm (no LP, QP or other formulation is required). The underlying principle of the method is the solution of transformed optimality conditions by means of the Newton-Raphson approach. This method combines ideas from both Class B.1 and Class B.2 OPF solution approaches.

Assume that the functional inequality constraints have been reformulated as equality constraints, (5) and (6). Solving (5) for the variables $\mathrm{z}$ and replacing them into the fourth optimality condition of (3) yields: 


$$
\begin{aligned}
\operatorname{diag}\{\widehat{\mu}\} \frac{\partial \mathcal{L}}{\partial \mu}=-\operatorname{diag}\{\widehat{\mu}\} \widehat{\mathbf{z}} & =\mathbf{0} \\
\widehat{\mu} & \geq \mathbf{0} \\
\widehat{\mathbf{z}} & \geq \mathbf{0}
\end{aligned}
$$

Optimality is reached only when among other conditions the $\mu_{i}$ and $z_{i}$ values are $\geq 0$. These conditions, however, can be eliminated by performing a variable transformation such that both the original $\mu_{i}$ and the original $z_{i}$ variables remain in the positive variable space. The fact that the $\mu_{i}$ and the $z_{i}$ variables must be positive or zero can be used to replace all occurrences of $\mu_{i}$ in the optimality conditions by positive expressions $\mu_{i}^{2 s}$ and all occurrences of $z_{i}$ by positive expressions $z_{i}^{2 r}$ :

$$
\mu_{i}^{\text {org }} \Rightarrow \mu_{i}^{2 s} \text { and } z_{i}^{\text {org }} \Rightarrow z_{i}^{2 r}
$$

with $s \neq 0$ and $r \neq 0$ ( $r$ and $s$ integer).

Applying these steps to the optimality conditions (3) the following transformed optimality conditions result:

$$
\begin{array}{ll}
\left.\frac{\partial}{\partial \mathbf{x}}\left(\mathcal{F}(\mathbf{x})+\widehat{\lambda}^{T} \mathbf{g}(\mathbf{x})+\widehat{\mu}^{2 s^{T}} \mathbf{h}(\mathbf{x})\right)\right|_{\hat{\mathbf{x}}} & =\mathbf{0} \\
\mathbf{g}(\widehat{\mathbf{x}}) & =\mathbf{0} \\
\mathbf{h}(\widehat{\mathbf{x}})+\widehat{\mathbf{z}}^{2 r} & =\mathbf{0} \\
\operatorname{diag}\left\{\widehat{\mu}_{i}^{2 s}\right\} \widehat{\mathbf{z}}^{2 r} & =\mathbf{0}
\end{array}
$$

The following problems have been solved with these transformed optimality conditions:

1. There is no explicit inequality in the optimality conditions.

2. The problem of maintaining feasibility during the solution process is eliminated. The individual transformed variables $\mu_{i}$ and $z_{i}$ are unlimited and can be either positive or negative during the iterations and also at the final solution.

3. Once a solution is obtained for the transformed optimality conditions the original values can be recomputed by $\mu_{i}^{\text {org }}:=\mu^{2 s}$ and $z_{i}^{\text {org }}:=z_{i}^{2 r}$.

It is not clear from a theoretical point of view what the best values for $r$ and $s$ are. Experience has shown that choosing $r=2$ and $s=1$ leads to good convergence for all tested networks. Other combinations for $r$ and $s$ have also been tested (see section "Simulation results"), however, overall, they have not shown the same algorithmic robustness for all tested networks.

The goal of this algorithm is the solution of the transformed optimality conditions as presented in (11). This solution is achieved by a simple Newton-Raphson algorithm as derived in the following subsection using no logarithmic barrier parameter and no step length control to stay within the feasible space (note: the feasible space is infinite for all variables). All known "tricks" to solve non-linear equations by a Newton-Raphson method can be used (for example, a damped Newton-Raphson).
2.2 Solution of transformed optimality conditions by Newton-Raphson

Applying a Newton-Raphson algorithm to (11) leads to the following main Newton-Raphson step which represents the solution of a huge sparse system of linear equations.

This linear system of equations represents one step in the Newton-Raphson solution process. The partial derivatives are with respect to all transformed problem variables, i.e. the variables $\mathbf{x}, \lambda, \mu$ and $\mathbf{z}$ are needed to get the linearized equations. The following system of equation results:

$$
\left[\begin{array}{cc|cc}
\mathbf{A}_{4} & \mathbf{0} & \mathbf{0} & \mathbf{A}_{3} \\
\mathbf{0} & \mathbf{Q} & \mathbf{A}_{1}^{T} & \mathbf{A}_{2}^{\prime} \\
\hline \mathbf{0} & \mathbf{A}_{1} & \mathbf{0} & \mathbf{0} \\
\mathbf{A}_{3}^{\prime} & \mathbf{A}_{2} & \mathbf{0} & \mathbf{0}
\end{array}\right] \cdot\left[\begin{array}{c}
\Delta \mathbf{z} \\
\Delta \mathbf{x} \\
\Delta \lambda \\
\Delta \mu
\end{array}\right]=-\left[\begin{array}{c}
\mathbf{b}_{3} \\
\mathbf{c}^{\prime} \\
\mathbf{b}_{1} \\
\mathbf{b}_{2}
\end{array}\right]
$$

with

$$
\begin{aligned}
\mathbf{Q} & =\left.\left(\frac{\partial^{2} \mathcal{F}(\mathbf{x})}{\partial \mathbf{x}^{2}}+\sum_{i=1}^{m}\left(\lambda_{i}^{o} \frac{\partial^{2} g_{i}(\mathbf{x})}{\partial \mathbf{x}^{2}}\right)+\sum_{i=1}^{p}\left(\mu_{i}^{o^{2 s}} \frac{\partial^{2} h_{i}(\mathbf{x})}{\partial \mathbf{x}^{2}}\right)\right)\right|_{\mathbf{x}^{o}} \\
\mathbf{c}^{\prime} & =\left.\frac{\partial \mathcal{F}(\mathbf{x})}{\partial \mathbf{x}}\right|_{x^{o}}+\left.\frac{\partial \mathbf{g}(\mathbf{x})^{T}}{\partial \mathbf{x}}\right|_{x^{o}} \lambda^{o}+\left.\frac{\partial \mathbf{h}(\mathbf{x})^{T}}{\partial \mathbf{x}}\right|_{x^{o}} \mu^{o^{2 s}} \\
\mathbf{A}_{1} & =\left.\frac{\partial \mathbf{g}(\mathbf{x})}{\partial \mathbf{x}}\right|_{x^{o}} \\
\mathbf{A}_{2} & =\left.\frac{\partial \mathbf{h}(\mathbf{x})}{\partial \mathbf{x}}\right|_{x^{o}} \\
\mathbf{A}_{2}^{\prime} & =\left.2 s \cdot \frac{\partial \mathbf{h}(\mathbf{x})^{T}}{\partial \mathbf{x}}\right|_{x^{o}} \operatorname{diag}\left(\mu_{i}^{o^{2 s-1}}\right) \\
\mathbf{A}_{3}^{\prime} & =2 r \cdot \operatorname{diag}\left(z_{i}^{o^{2 r-1}}\right) \\
\mathbf{A}_{3} & =2 s \cdot \operatorname{diag}\left(\mu_{i}^{o^{2 s-1}} z_{i}^{o^{2 r}}\right) \\
\mathbf{A}_{4} & =2 r \cdot \operatorname{diag}\left(\mu_{i}^{o{ }^{2 s}} z_{i}^{\alpha^{2 r-1}}\right) \\
\mathbf{b}_{1} & =\mathbf{g}\left(\mathbf{x}^{o}\right) \\
\mathbf{b}_{2} & =\mathbf{h}\left(\mathbf{x}^{o}\right)+\mathbf{z}^{o^{2 r}} \\
\mathbf{b}_{3} & =\operatorname{diag}\left(z_{i}^{o^{2 r}}\right) \mu^{o^{2 s}}
\end{aligned}
$$

An upper index of ${ }^{o}$ indicates a numerically given variable of the iteration. In this system of equations (13) certain variables can be easily eliminated (e.g. $\Delta z$ and also $\Delta \mu$ ). This would lead to a much smaller linear system. However, the main issue of this paper is to explain the concept and not algorithmic speed-up efforts.

This sparse linear system of equations can be solved by any robust sparse linear system solver algorithm. In the actual computer implementation a public-domain package [16] is used. The resulting $\Delta$ variables are updated as done in the conventional Newton-Raphson algorithm and then the new linear system of equations is solved again.

Comparing this algorithm to the previously discussed methods, the main Newton-Raphson step of this method has many similarities with the primal-dual interior-point algorithms. The main difference is the non-existence of either a feasibility region based step-control or a barrier parameter $\zeta$. Also, no logarithmic barrier objective function is needed in the derivation of the approach. When comparing the matrices to the class B.1 algorithms many almost identical terms can be detected. The main difference, is, that no heuristic tuning of weights and no step 


\begin{tabular}{|c|c|c|c|c|c|c|c|c|c|c|c|c|c|c|}
\hline buses & $\begin{array}{l}\text { load } \\
\text { MW }\end{array}$ & $\begin{array}{r}\text { load } \\
\text { MVar }\end{array}$ & $\begin{array}{r}\text { bran- } \\
\text { ches }\end{array}$ & $\begin{array}{l}\text { vars } \\
\mathrm{x}, \lambda\end{array}$ & $\begin{array}{l}\text { inequ. } \\
\text { init. }\end{array}$ & $\begin{array}{r}\text { inequ. } \\
\text { final }\end{array}$ & $\alpha$ & $\begin{array}{c}\text { VMax } \\
\text { bind. }\end{array}$ & $\begin{array}{c}\text { QMax } \\
\text { bind. }\end{array}$ & $\begin{array}{l}\text { QMin } \\
\text { bind. }\end{array}$ & $\begin{array}{r}\text { Loss } \\
\text { initial }\end{array}$ & $\begin{array}{l}\text { Loss } \\
\text { final }\end{array}$ & iter. & $\begin{array}{r}\mathrm{CPU} \\
\mathrm{sec}\end{array}$ \\
\hline 14 & 259 & 74 & 20 & 71 & 34 & 36 & 0.8 & 1 & 1 & 0 & 13.4 & 13.3 & 11 & 0.43 \\
\hline 24 & 2850 & 1580 & 38 & 123 & 58 & 60 & 1.0 & 6 & 2 & 0 & 51.6 & 45.9 & 12 & 0.55 \\
\hline 30 & 283 & 126 & 41 & 127 & 61 & 66 & 1.0 & 2 & 1 & 0 & 17.6 & 17.5 & 11 & 0.62 \\
\hline 41 & 1950 & 877 & 68 & 262 & 140 & 142 & 1.0 & 5 & 2 & 0 & 24.7 & 21.2 & 11 & 1.09 \\
\hline 57 & 1251 & 336 & 80 & 236 & 114 & 122 & 1.0 & 0 & 2 & 0 & 27.9 & 25.7 & 11 & 1.04 \\
\hline 73 & 8550 & 1740 & 120 & 365 & 172 & 178 & 1.0 & 23 & 6 & 0 & 158 & 133 & 13 & 1.75 \\
\hline 118 & 3668 & 1438 & 186 & 527 & 239 & 280 & 1.0 & 7 & 10 & 3 & 132.5 & 111.6 & 19 & 3.53 \\
\hline 685 & 109521 & 20659 & 1392 & 2935 & 1390 & 1482 & 0.8 & 76 & 35 & 5 & 1393.7 & 1247.5 & 25 & 39.4 \\
\hline
\end{tabular}

Table 1: Key values for test networks

lenghts reduction due to feasibility space restrictions are necessary.

The main feature of the algorithm is its unlimited variable space which leads to the name Unlimited Point (UP) algorithm (in contrast to interior point (IP) algorithms, which have a limited interior variable space).

\subsection{Summary of algorithmic steps}

Solving the original linear system of equations leads to the following steps:

1. Choose an initial starting value for the variables $\mathbf{x}^{o}, \lambda^{o}, \mu^{o}, \mathbf{z}^{o}$. Set $r=2$ and $s=1$.

2. Solve the sparse linear system of equations (12).

3. Update all variables using step length control to allow better Newton-Raphson convergence:

$$
\begin{array}{ll}
\mu^{o} & :=\mu^{o}+\alpha \Delta \mu \\
\lambda^{o} & :=\lambda^{o}+\alpha \Delta \lambda \\
\mathbf{x}^{o} & :=\mathbf{x}^{o}+\alpha \Delta \mathbf{x} \\
\mathbf{z}^{o} & :=\mathbf{z}^{o}+\alpha \Delta \mathbf{z}
\end{array}
$$

The factor $\alpha$ serves only the purpose of damping the Newton-Raphson algorithm. It depends on network characteristics as shown in the simulation results section.

4. If all absolute values of the right hand sides of the linear system (12) are below a given tolerance STOP, otherwise go to step 2.

\section{Simulation results}

The concepts as presented in section 2 are mathematically straightforward. However, their implementation leads to several practical problems. One problem is the inclusion of all possible inequality constraints from the beginning into the problem formulation. Due to the fact that only a few inequality constraints become binding the computational effort of including all constraints is much too high. Also, simulation results have shown that for certain networks convergence robustness degrades if too many "inactive" inequality constraints are included in the actual solution process. This problem has been avoided as follows:

First, a power flow is executed using the same algorithm, however, with no degree of freedom. Next, the iterative OPF solution process is started with a set of "critical" inequality constraints which includes all lower and upper voltage magnitude limits and the violated reactive power limits. With this set of inequality constraints one NR iteration is executed (using reactive generator powers, generator voltage magnitudes and transformer taps as controls), new violated reactive power limits are added, one NR iteration is executed, new violated reactive power limits are added, etc. until final convergence is obtained with no limit violations both on voltages and reactive powers.

This algorithm has been tested with a variety of test networks using total active power losses as objective function. Test networks have been IEEE 14, 30, 57, 118 [17], 24, 41, 73 bus CIGRE networks and a 685 bus network. The convergence criterion was $0.01 \mathrm{p}$.u. on a $100 \mathrm{MVA}$ basis. All runs have been executed with a predefined starting value for both $\mu_{i}$ and $z_{i}$. All runs had zero binding lower voltage limits. The lower voltage limit is throughout all runs 0.90 p.u., the upper voltage limit at all buses is 1.10 p.u. Table 1 summarizes the main OPF convergence characteristics of these networks.

In Table 1, the columns have the following meaning in order: Total number of buses; total active/reactive load power; total number of branches (lines and transformers); number of variables $x$ and $\lambda$ (i.e. the total number of unknowns $x$ and number of equality constraints $g(x)$ ); initial number of inequality constraints after a regular power flow; final number of inequality constraints at the last OPF iteration; the Newton-Raphson damping parameter $\alpha$ (identical for all iterations); number of binding inequality constraints at bus maximum voltage and at generator bus reactive power maximum; at generator bus reactive power minimum; active power losses in MW after the initial power flow (i.e. the initial losses of the optimization); active power losses in MW at the optimum; total CPU time in seconds on a $167 \mathrm{MHz}$ Sun Ultra Sparc (including data read phase and print-out of important parameters).

Table 2 compares the solution accuracy of the obtained OPF solutions with a run with an extreme choice $r=$ $5, s=5$ with the most robust choice of $r=2, s=1$.

\begin{tabular}{r|r} 
variable type & $\mathrm{r}=5, \mathrm{~s}=5$ \\
\hline $\mathrm{x}$ & 0.0008031 \\
$\mu^{(2 r)}$ & 0.00002873 \\
$z^{(2 s)}$ & 0.0043
\end{tabular}

Table 2: OPF Solution accuracy comparison for $r=5, s=$ 5 as compared to $r=2, s=1$ for the 118 bus system 
In Table 2 the value in the second column indicates the maximum absolute difference of each type of variable category. One can notice only a slight difference in all three categories of variables (x: state/control variables, $\mu$ : Lagrange multipliers for inequality constraints, $z$ : slack variables of inequality constraints). This high accuracy has been confirmed in all cases where the optimal objective function value was numerically close to the reference optimal power flow $(r=2, s=1)$ objective function value.

Tables 3 and 4 show different convergence behavior for the $118 / 685$ bus networks with varying $r, s$ and starting values for $\mu$ and $z$.

\begin{tabular}{r|r|r|r|r|r}
$\mathrm{r}$ & $\mathrm{s}$ & $\mu_{\text {start }}$ & $z_{\text {start }}$ & $\alpha$ & iter \\
\hline 1 & 1 & 1.0 & 1.0 & 1.0 & 19 \\
1 & 2 & 1.0 & 1.0 & 1.0 & 19 \\
2 & 1 & 1.0 & 1.0 & 1.0 & 19 \\
2 & 1 & 1.0 & 1.0 & 0.8 & 22 \\
2 & 2 & 1.0 & 1.0 & 1.0 & 16 \\
3 & 3 & 1.0 & 1.0 & 1.0 & 16 \\
2 & 3 & 1.0 & 1.0 & 1.0 & 16 \\
5 & 5 & 1.0 & 1.0 & 1.0 & 17 \\
10 & 10 & 1.0 & 1.0 & 1.0 & 18 \\
25 & 25 & 1.0 & 1.0 & 1.0 & 18 \\
2 & 1 & 0.5 & 0.5 & 1.0 & 12 \\
2 & 1 & 0.2 & 0.2 & 1.0 & - \\
2 & 1 & 1.5 & 1.5 & 1.0 & - \\
2 & 1 & 0.5 & 1.5 & 1.0 & 16 \\
2 & 1 & 1.5 & 0.5 & 1.0 & 12 \\
2 & 1 & 1.1 & 1.1 & 1.0 & 24 \\
2 & 2 & 0.5 & 0.5 & 1.0 & -
\end{tabular}

Table 3: Convergence with different $r, s$ and starting values for $\mu, z$-variables for 118 bus system

\begin{tabular}{r|r|r|r|r|r}
$\mathrm{r}$ & $\mathrm{s}$ & $\mu_{\text {start }}$ & $z_{\text {start }}$ & $\alpha$ & iter \\
\hline 1 & 2 & 0.8 & 0.8 & 0.8 & 29 \\
1 & 2 & 0.8 & 0.8 & 1.0 & - \\
1 & 2 & 1.0 & 1.0 & 0.8 & 25 \\
2 & 2 & 1.0 & 1.0 & 0.8 & 27 \\
2 & 2 & 0.8 & 0.8 & 1.0 & - \\
2 & 2 & 0.8 & 0.8 & 0.8 & 23 \\
2 & 1 & 0.8 & 0.8 & 0.8 & 23 \\
2 & 1 & 1.0 & 1.0 & 0.8 & 25 \\
2 & 1 & 0.5 & 0.5 & 0.8 & - \\
2 & 1 & 1.0 & 1.0 & 1.0 & - \\
3 & 3 & 1.0 & 1.0 & 1.0 & - \\
3 & 3 & 0.8 & 0.8 & 1.0 & - \\
3 & 3 & 0.8 & 0.8 & 0.8 & 26
\end{tabular}

Table 4: Convergence with different $r, s$ and starting values for $\mu, z$-variables for 685 bus system

In Tables 3 and 4 the sign "_" in the last column indicates a non-converged case. One concludes that a) with values of $\alpha<1$ the convergence is usually slower than with $\alpha=1$ (if this case converges), but for the large network no convergence has been obtained with $\alpha=1$, b) the best choice of $r, s$ for the 118 bus network $(r=2, s=1)$ is not the best for the 685 bus network (here, the best combination is $r=2, s=1$ ), c) the number of iterations depends on the initial value chosen for $\mu$ and $z$ : The best initial values for $\mu$ and $z$ for the 118 bus network are 0.5 for both variables. With these values, no convergence is obtained for the large 685 bus network where the best starting values for $\mu$ and $z$ are 0.8 .
Tables 5 and 6 show the complete right-hand-side mismatch convergence for the 118 bus network for different choices of $r$ and $s$. The columns indicate: NewtonRaphson iteration number; maximum mismatch of the total right hand side; maximum absolute mismatch of $c^{\prime}$ of (13) ; maximum absolute mismatch in (13) of $b_{1}$, of $b_{2}$, of $b_{3}$; value of the objective function; number of upper and lower reactive power inequality constraints added to the original set of inequality constraints.

Comparing these two tables 5 and 6 , Table 6 shows overall faster convergence $(r=2$ and $s=2)$. However, in general the convergence after iteration 9 is not as monotonic as with $r=2$ and $s=1$ (Table 5).

The key point of the algorithm is that convergence is obtained without limits on any transformed variables. Table 7 shows the convergence of transformed $\mu_{i}$, transformed $z_{i}$ and the corresponding untransformed $\mu_{i}^{2 s}(\mathrm{~s}=1)$ and $z_{i}^{2 r}$ $(\mathrm{r}=2)$ for three selected upper bus voltage limit inequality constraints.

The buses and the related inequality constraints shown are of a different nature: The first eight columns in Table 7 show the convergence of these values for the limited quantity $V_{\text {Max }}$ at bus 37 and $V_{\text {Max }}$ at bus 4 . These two inequality constraints become binding at the optimum. The next four columns represent the convergence of these values for the limited quantity $V_{M a x}$ at bus 1 . This limit does not become binding in the optimum.

The first two binding inequality constraints behave differently: In the first case, the $z_{i}$ value tends towards a slightly negative value, whereas in the second case, the value $z_{i}$ becomes slightly positive.

One of the key feature of convergence is shown with the plot of the original, untransformed variables $\mu_{i}^{2 s}(\mathrm{~s}=1)$ and $z_{i}^{2 r}(\mathrm{r}=2)$ : Whenever final convergence is approached, one of the values of $\mu_{i}^{2 s}(\mathrm{~s}=1)$ and $z_{i}^{2 r}(\mathrm{r}=2)$ tends towards zero for each inequality $i$. The original variables $\mu_{i}^{2 s}(\mathrm{~s}=1)$ and $z_{i}^{2 r}(\mathrm{r}=2)$ will be much nearer to zero than the untransformed, original variables (for example $0.1^{4}=0.0001$ ). This effect is also clearly shown in the third inequality of Table 7 where the transformed $\mu_{i}$-values goes near, but not very near to zero. The untransformed, original $\mu_{i}^{2 s}$ $(\mathrm{s}=1)$, however, is very near to zero at the optimal solution.

\section{Conclusions}

In this paper the KKT-optimality conditions are transformed to a set of optimality conditions represented by a well determined set of equations. All inequality constraints are eliminated. This is achieved by transforming all occurrences of variables which must be positive in the optimum to expressions of new variables which can only be positive for real variables. These new variables, however, are unlimited in the space of real numbers. The algorithm to solve this transformed set of non-linear equations is the standard Newton-Raphson algorithm. Once the solution is obtained the original variables can easily be obtained by applying the transformation rule again. 


\begin{tabular}{|c|c|c|c|c|c|c|c|}
\hline iter & $\max \mid$ mism $\mid$ & $\max \left|\frac{\partial \mathcal{L}}{\partial x}\right|$ & $\max |g(x)|$ & $\max \left|h(x)+z^{2 r}\right|$ & $\max \left|\mu^{2 s} z^{2 r}\right|$ & Obj. funct. & Added $Q_{L i m}$ \\
\hline 0 & 1.048862 & 1.000000 & 0.000000 & 1.048862 & 1.000000 & 132.477796 & 3 \\
\hline 1 & 2.245746 & 1.000059 & 0.058251 & 2.245746 & 1.000000 & 124.361194 & 13 \\
\hline 2 & 2.986861 & 1.000082 & 0.073632 & 2.986861 & 1.000000 & 136.705072 & 18 \\
\hline 3 & 1.678159 & 0.999994 & 0.112308 & 1.678159 & 1.000000 & 128.977803 & 9 \\
\hline 4 & 0.532482 & 0.328177 & 0.046153 & 0.532482 & 0.321956 & 122.973896 & 0 \\
\hline 5 & 0.107475 & 0.062667 & 0.006845 & 0.087566 & 0.107475 & 119.914874 & 0 \\
\hline 6 & 0.043922 & 0.016409 & 0.014647 & 0.043922 & 0.032714 & 114.576984 & 0 \\
\hline 7 & 1.752327 & 1.000000 & 0.011372 & 1.752327 & 1.000000 & 110.864630 & 1 \\
\hline 8 & 0.253975 & 0.242033 & 0.172157 & 0.102098 & 0.253975 & 112.817323 & 0 \\
\hline 9 & 0.215061 & 0.058093 & 0.002910 & 0.215061 & 0.066634 & 112.653309 & 0 \\
\hline 10 & 0.051422 & 0.012390 & 0.001778 & 0.051422 & 0.019119 & 112.377928 & 0 \\
\hline 11 & 0.092381 & 0.003050 & 0.004135 & 0.092381 & 0.006174 & 112.017367 & 0 \\
\hline 12 & 0.097781 & 0.047281 & 0.003410 & 0.097781 & 0.002067 & 111.736411 & 0 \\
\hline 13 & 0.099089 & 0.004718 & 0.000601 & 0.099089 & 0.000674 & 111.633570 & 0 \\
\hline 14 & 0.111323 & 0.000074 & 0.000020 & 0.111323 & 0.000215 & 111.615073 & 0 \\
\hline 15 & 0.514410 & 0.000037 & 0.000000 & 0.514410 & 0.000068 & 111.613834 & 0 \\
\hline 16 & 0.190450 & 0.000008 & 0.000001 & 0.190450 & 0.000022 & 111.613840 & 0 \\
\hline 17 & 0.095243 & 0.000014 & 0.000002 & 0.095243 & 0.000007 & 111.613886 & 0 \\
\hline 18 & 0.023291 & 0.000799 & 0.000121 & 0.023291 & 0.000002 & 111.616175 & 0 \\
\hline 19 & 0.003836 & 0.000066 & 0.000015 & 0.003836 & 0.000001 & 111.617817 & 0 \\
\hline
\end{tabular}

Table 5: Complete right-hand-side convergence for 118 bus system with $r=2$ and $s=1$

\begin{tabular}{r|r|r|r|r|r|r|r|} 
iter & $\max \mid$ mism $\mid$ & $\max \left|\frac{\partial \mathcal{L}}{\partial x}\right|$ & $\max |g(x)|$ & $\max \left|h(x)+z^{2 r}\right|$ & $\max \left|\mu^{2 s} z^{2 r}\right|$ & Obj. funct. & Added $Q_{\text {Lim }}$ \\
\hline 0 & 1.048862 & 1.000000 & 0.000000 & 1.048862 & 1.000000 & 132.477796 & 3 \\
1 & 2.245746 & 1.000059 & 0.058251 & 2.245746 & 1.000000 & 124.361194 & 13 \\
2 & 2.987431 & 1.000082 & 0.073547 & 2.987431 & 1.000000 & 136.605896 & 18 \\
3 & 1.677710 & 0.999995 & 0.113463 & 1.677710 & 1.000000 & 128.967735 & 9 \\
4 & 0.486329 & 0.362724 & 0.041211 & 0.486329 & 0.343460 & 123.043621 & 0 \\
5 & 0.117928 & 0.100157 & 0.004249 & 0.078378 & 0.117928 & 120.924000 & 0 \\
6 & 0.039675 & 0.032622 & 0.007666 & 0.014498 & 0.039675 & 116.945951 & 0 \\
7 & 1.270745 & 1.000000 & 0.013028 & 1.270745 & 1.000000 & 112.369461 & 1 \\
8 & 0.317927 & 0.308006 & 0.114918 & 0.167779 & 0.317927 & 112.810183 & 0 \\
9 & 0.101939 & 0.094428 & 0.002788 & 0.043394 & 0.101939 & 112.779141 & 0 \\
10 & 0.038964 & 0.027215 & 0.000631 & 0.038964 & 0.033370 & 112.492267 & 0 \\
11 & 0.035279 & 0.006950 & 0.002885 & 0.035279 & 0.011276 & 112.181051 & 0 \\
12 & 0.078492 & 0.007505 & 0.003591 & 0.078492 & 0.003874 & 111.866534 & 0 \\
13 & 0.086195 & 0.036247 & 0.001691 & 0.086195 & 0.001304 & 111.684736 & 0 \\
14 & 0.092339 & 0.007016 & 0.000206 & 0.092339 & 0.000422 & 111.622899 & 0 \\
15 & 0.050182 & 0.008497 & 0.000131 & 0.050182 & 0.000252 & 111.619031 & 0 \\
16 & 0.005529 & 0.002344 & 0.000062 & 0.005529 & 0.000082 & 111.620598 & 0
\end{tabular}

Table 6: Complete right-hand-side convergence for 118 bus system with $r=2$ and $s=2$

\begin{tabular}{|c|c|c|c|c|c|c|c|c|c|c|c|c|}
\hline It & \multicolumn{4}{|c|}{$V_{\text {Max }}$ at bus 37} & \multicolumn{4}{|c|}{$V_{\text {Max }}$ at bus 4} & \multicolumn{4}{|c|}{$V_{\text {Max }}$ at bus 1} \\
\hline & $\mu_{i}$ & $z_{i}$ & $\mu_{i}^{2}$ & $z_{i}^{4}$ & $\mu_{i}$ & $z_{i}$ & $\mu_{i}^{2}$ & $z_{i}^{4}$ & $\mu_{i}$ & $z_{i}$ & $\mu_{i}^{2}$ & $z_{i}^{4}$ \\
\hline 1 & 0.9175 & 0.7913 & 0.84178 & 0.39198554 & 0.9116 & 0.7942 & 0.83103 & 0.39784119 & 0.89781 & 0.8011 & 0.80605955 & 0.41185 \\
\hline 2 & 0.7314 & 0.6737 & 0.53493 & 0.20598740 & 0.7309 & 0.6744 & 0.53418 & 0.20682632 & 0.54319 & 0.7590 & 0.29505547 & 0.33192 \\
\hline 4 & 0.1960 & 0.6544 & 0.03842 & 0.18341071 & 0.2291 & 0.6337 & 0.05250 & 0.16121387 & 0.16960 & 0.7000 & 0.02876504 & 0.24008 \\
\hline 5 & 0.1140 & 0.6278 & 0.01299 & 0.15532594 & 0.1251 & 0.6191 & 0.01564 & 0.14692268 & 0.08962 & 0.6900 & 0.00803185 & 0.22673 \\
\hline 6 & 0.0800 & 0.5644 & 0.00640 & 0.10148836 & 0.0854 & 0.5625 & 0.00730 & 0.10008534 & 0.05623 & 0.6461 & 0.00316182 & 0.17424 \\
\hline 9 & 0.0373 & 0.3573 & 0.00139 & 29201 & 0.0715 & 0.2651 & 0.00512 & 3729 & 0.01479 & 0.5147 & 21883 & 0.07018 \\
\hline 10 & 0.0360 & 0.2741 & 0.00129 & 0.00564607 & 0.0939 & 0.1573 & 0.00882 & 0.00061257 & 0.00791 & 0.5057 & 0.00006265 & 0.06539 \\
\hline 11 & 0.0539 & 0.1374 & 0.00290 & 0.00035679 & 0.1321 & 0.0860 & 0.01745 & 0.00005474 & 0.00387 & 0.5084 & 0.00001500 & 0.06679 \\
\hline 12 & 0.2026 & -0.0867 & 0.04105 & 0.00005657 & 0.1227 & 0.0676 & 0.01504 & 0.00002087 & 0.00192 & 0.5096 & 0000368 & 0.06744 \\
\hline 13 & 0.1558 & -0.0751 & 0.02 & & & 0.0 & & & & & 992 & 747 \\
\hline 14 & 0.1502 & -0.0577 & 0.02255 & 0.00001105 & 0.1213 & 0.0383 & 0.01472 & 0.00000215 & 0.00048 & 0.5097 & 0.00000023 & 0.06747 \\
\hline 15 & 0.1502 & -0.0432 & 0.02257 & 0.00000349 & 0.1213 & 0.0287 & 0.01471 & 0.00000068 & 0.00024 & 0.5097 & 0.00000006 & 0.06747 \\
\hline
\end{tabular}

Table 7: Convergence for selected transformed and untransformed variables for 118 bus system $(r=2, s=1)$ 
This Unlimited Point method has many similarities with the Newton-approach. It is also conceptually very similar to interior point algorithms. Its main advantage is, however, that its solution process is neither based on heuristics for the determination of binding inequality constraints, nor is it required to have intermediate feasible solutions.

The method has been applied to various networks and performance results indicate the same properties as published for interior points methods: the computational time increases only linearly with network problem size.

The Unlimited Point algorithm is very general and could be applied to any smooth non-linear optimization problem. However, in this paper, the method is successfully applied only to the OPF domain. The application of the method to other optimization domains remains to be investigated.

\section{REFERENCES}

[1] H.W.Kuhn and A.W.Tucker. Non-linear programming. Proc. 2nd Berkeley Symposium on Mathematics, Statistics and Probability, University of California Press, Berkeley, California, 1951.

[2] H. Glavitsch and R. Bacher. Optimal Power Flow Techniques for Electric Power Systems. Academic Press, 1991.

[3] K.Frauendorfer, H. Glavitsch, and R. Bacher (Eds). Optimization in Planning and Operation of Electric Power Systems. Physica Verlag, Springer, 1993.

[4] D.I. Sun, B. Ashley, B. Brewer, A. Hughes, and W.F. Tinney. Optimal power flow by newton approach. IEEE Transactions on Power Apparatus and Systems, (10):2864-2880, Oct, 1984

[5] N.K. Karmarkar. A new polynomial time algorithm for linear programming. Combinatorica, 4, pages 373-395, 1984 .

[6] N.K. Karmarkar. Computational results of an interior point algorithm for large scale linear programming. Mathematical Programming, 52, pages 555-586, 1991.

[7] M. Kojima, N. Megiddo, T. Noma, and A. Yoshise. Unified Approach to Interior Point Algorithms for Linear Complimentarity Problems. Springer-Verlag Berlin, 1991.

[8] R. W. Cottle, J-S. Pang, and R. Stone. The linear complementarity problem. Academic Press, Inc., San Diego, 1992.

[9] H. Wei, H. Sasaki, and R. Yokoyama. An application of interior point quadratic programming algorithm to power system optimization problems. IEEE Transactions on Power Systems, 1(1):260-266, Feb, 1996.

[10] S. Granville. Optimal reactive dispatch through interior point methods. IEEE Transactions on Power Systems, 9(1):136-146, Feb, 1994.

[11] J.A. Momoh, S.X. Guo, E.C. Ogbuobiri, and R. Adapa. The quadratic interior point method solving power system optimization problems. IEEE Transactions on Power Systems, 9(3):1327-1336, Aug, 1994.

[12] Y.C. Wu, A.S. Debs, and R.E. Marsten. A direct nonlinear predictor-corrector primal-dual interior point algorithm for optimal power flow. IEEE Transactions on Power Systems, 9(2):876-883, May, 1994.
[13] G.D. Irisarri, X.Wang, J.Tong, and S.Mokhtari. Maximum loadability of power systems using interior point non-linear optimization method. IEEE Winter Power Meeting Jan. 1996, Baltimore, 1996. 96 WM 207-1 PWRS.

[14] G.Blanchon, J.C.Dodu, A.Renaud, and M.Bouhtou. Implementation of a primal-dual interior-point method applied to the planning of reactive power compensation. 12th PSCC96 Power Systems Computations Conference, pages 827-836, Aug, 1996.

[15] J.L. Martinez Ramos, A. Gomez Exposito, and V.H.Quintana. Reactive-power optimization by interiorpoint methods: Implementation issues. 12th PSCC96 Power Systems Computations Conference, pages 844-850, Aug, 1996.

[16] Timothy A. Davis and Iain S. Duff. Umfpack version 2.0: Unsymmetric-pattern multifrontal package. Sept, 1995. Program package at: http://www.cis.ufl.edu/ davis.

[17] University of washington energy group power systems archive. ftp://wahoo.ee.washington.edu/.

Giorgio Tognola received the Dipl.El.-Ing. degree in electrical engineering in 1992 from the Swiss Federal Institute of Technology (ETH) in Zürich, Switzerland. He is currently finishing his $\mathrm{PhD}$ in the area of power system optimization.

Rainer Bacher received the Dipl.El.-Ing. degree in electrical engineering in 1982 and the Dr.sc.techn. degree in 1986, both from the Swiss Federal Institute of Technology (ETH) in Zürich, Switzerland. After his doctorate he joined the Energy Management Systems Division of Control Data Corporation in Minneapolis, U.S.A. In 1989 he joined Colenco Ltd., a Swiss consulting company. Also in 1989 he started as a lecturer and part-time senior researcher at the power system group at the ETH. In 1992 he became a full-time senior assistant and lecturer at the ETH. In 1993 he was appointed assistant professor of Energy Management Systems at the department of electrical engineering at the ETH Zürich. He is a member of technical committees for PSCC 93, 96 and PICA 93, 95, 97. He can be reached at Internet e-mail: Rainer.Bacher@eeh.ee.ethz.ch. His WWW home page is http://www.eus.ee.ethz.ch/ ${ }^{\text {bacher. }}$ 